\title{
Transmission of an FMR1 premutation allele in a large family identified through newborn screening: the role of AGG interruptions
}

\author{
Carolyn M Yrigollen ${ }^{1}$, Guadalupe Mendoza-Morales ${ }^{1}$, Randi Hagerman ${ }^{2,3}$ and Flora Tassone ${ }^{1,2}$
}

The CGG repeat within the premutation range in the fragile $\mathrm{X}$ mental retardation 1 (FMR1) gene can lead to neurodegenerative disorders and intellectual disabilities. An increase in size upon the transmission from parent to child is more likely to occur for larger alleles and without AGG interruptions. We describe the molecular structure and the transmission of an FMR1 premutation allele in a multigenerational family, identified through newborn screening for fragile $X$ syndrome. Transmission of the premutation allele was traced through five generations in 14 of the 23 individuals who were genotyped through cascade testing. Allele size instability during transmission was observed, but no expansions to a full mutation were detected. Clinical and molecular characterizations of the participants lead to the diagnosis of fragile $X$-associated tremor ataxia syndrome in one subject identified as a premutation carrier. A gradual small increase in the size of the premutation allele was observed during transmission through five generations. The relative stability is likely due to the presence of two AGGs within the allele.

The detection of AGG interruptions within the premutation alleles is important in genetic counseling, to better predict the risk of expansion during transmission from a premutation to a full-mutation allele.

Journal of Human Genetics (2013) 58, 553-559; doi:10.1038/jhg.2013.50; published online 6 June 2013

Keywords: AGG interruptions; FMR1; newborn screening; premutation

\section{INTRODUCTION}

The fragile $\mathrm{X}$ mental retardation 1 (FMR1) gene is essential for normal development. ${ }^{1}$ Mutations in the FMR1 gene are causative of a number of different genetic disorders, including fragile X syndrome (FXS), fragile X-associated tremor/ataxia syndrome (FXTAS), and fragile $\mathrm{X}$-associated primary ovarian insufficiency (FXPOI) ${ }^{2,3}$ Besides these distinct disorders, many other clinical phenotypes have been associated with the FMR1 gene, including increased risk of autism spectrum disorders, attention deficit hyperactivity disorder, anxiety and depression. $^{4-7}$

Greater than 200 CGG repeats in the FMR1 gene is defined as a full mutation and leads to FXS. The long GC-rich stretch of DNA becomes hypermethylated and silences transcription of the gene resulting in a loss of the FMR1 protein. ${ }^{8,9}$ FXTAS and FXPOI are known to occur in individuals who carry a premutation allele (55-200 repeats), both have a late onset, and it is currently thought that they are the result of an RNA toxicity mechanism. ${ }^{10,11}$ Expanded FMR1 mRNA, which are elevated in premutation carriers, ${ }^{12-14}$ are thought to sequester proteins from their cellular function, including Pur $\alpha$, hnRNP A2/B1 and Sam68. ${ }^{15-18}$

Within the CGG repeat tract of FMR1 are cytosine to adenine transversions, which interrupt stretches of CGG elements with an
AGG. ${ }^{19}$ AGG interruptions occur in normal, intermediate and premutation range alleles. Common patterns emerge when interrogating the locus for the presence and location of AGG interruptions (that is, (CGG) 9 AGG (CGG) $)_{9}$ AGG (CGG) $)_{9}$, although their frequency fluctuates, in different populations. ${ }^{20,21}$ AGG interruptions are thought to stabilize the CGG repeat tract during transmission, protecting against expansion ${ }^{22,23}$, but do not influence either the transcription or the translation of the FMR1 gene. ${ }^{12,24,25}$

A recent PCR assay has been established that allows rapid detection of premutation and full-mutation alleles. ${ }^{26-28}$ Using this assay a multisite (at UC Davis Medical Center (Sacramento, CA, USA), Rush University Medical Center (RUMC, Chicago, IL, USA) and the University of North Carolina Hospital (UNC, Chapel Hill, NC, USA)) newborn screening study was conducted in order to accurately estimate the allele frequencies of the FMR1 expanded alleles. ${ }^{29}$ The recruitment protocol for this study allows researchers to approach the families of individuals identified as carriers of a premutation or full-mutation allele for clinical services, follow up evaluations and perform cascade testing.

One of the newborns identified during the screening was a male carrier of a premutation allele of 68 CGG repeats (Figure 1a)

${ }^{1}$ Department of Biochemistry and Molecular Medicine, University of California Davis, School of Medicine, Davis, CA, USA; ${ }^{2}$ M.I.N.D. Institute, University of California Davis Medical Center, Davis, CA, USA and ${ }^{3}$ Department of Pediatrics, University of California Davis Medical Center, Davis, CA, USA

Correspondence: Professor F Tassone, Department of Biochemistry and Molecular Medicine, University of California Davis, School of Medicine, 2700 Stockton Blvd., Suite 2102, Sacramento, CA 95817, USA.

E-mail: ftassone@ucdavis.edu

Received 22 January 2013; revised 2 April 2013; accepted 21 April 2013; published online 6 June 2013 

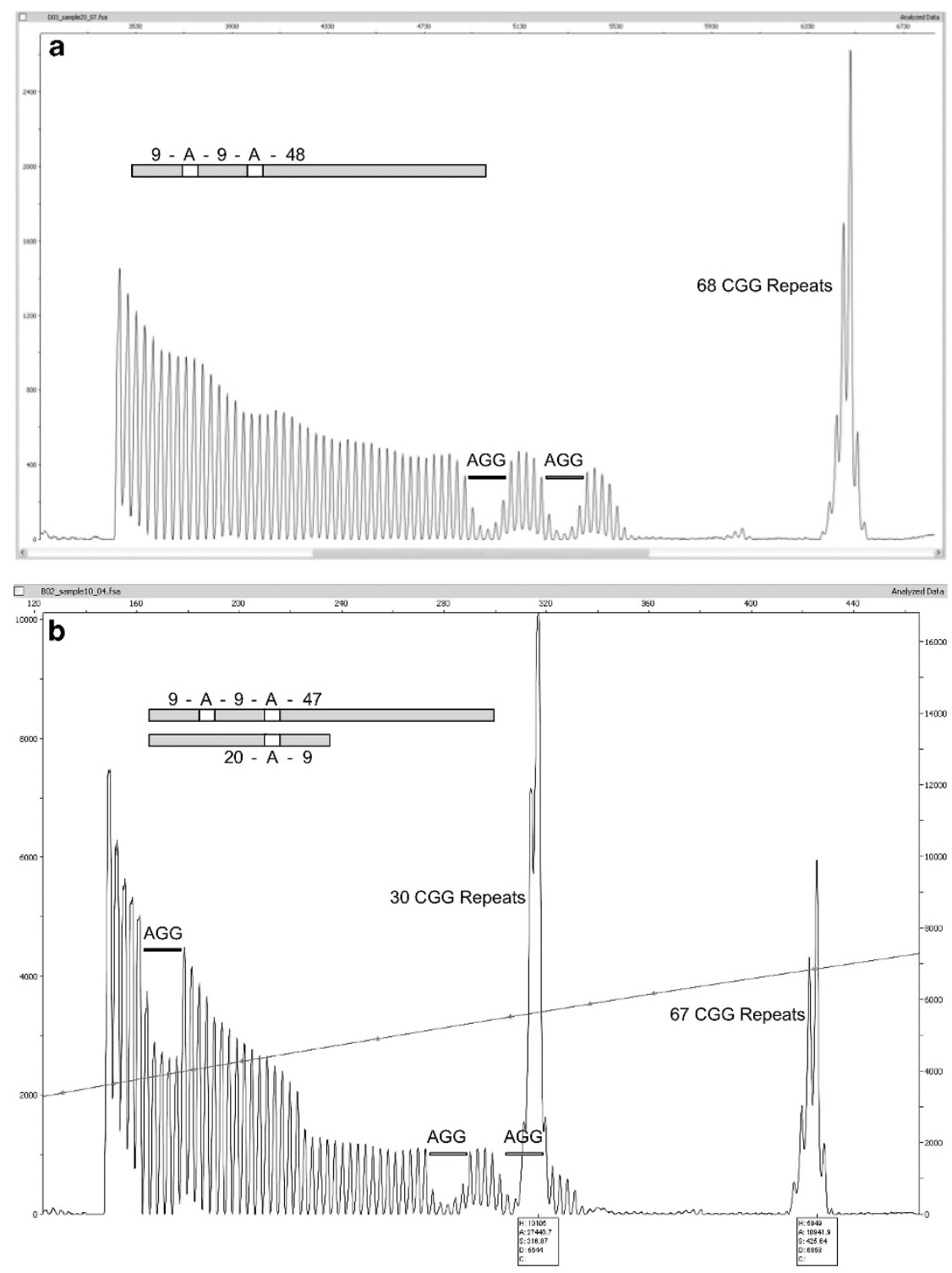

Figure 1 AGG interruption patterns determined by capillary electrophoresis analysis. The presence of AGG interruptions is visible on electropherogram after PCR amplification in one male and one female premutation carriers. (a) The proband, a male with 68 CGG repeats, has two AGG interruptions. (b) A female (V-10) with 67 CGG repeats in the premutation allele and 30 CGG repeats in the normal allele has two AGG interruptions.

(pedigree ID: V-11). The family of this individual was contacted for participation in genetic counseling services along with cascade testing to identify additional members of the family carrying an expanded allele. The CGG allele size was determined for 23 individuals within the family. The lineage of the premutation allele was traced from the proband's great grandmother to the great-great-grandfather. Here, we report on our findings of the stability of the premutation allele in 14 transmissions, and present the clinical findings of individuals who carry the inherited premutation allele.

\section{MATERIALS AND METHODS}

\section{Genetic screening}

The proband was screened as part of a pilot newborn screening study for mutations in the FMR1 CGG repeat locus. ${ }^{29}$ The screening was performed using protocols approved by the Institutional Review Board, and consent was obtained from all individuals participating in the study. Blood was collected on FTA bloodspot collection cards, amplification of the CGG repeat region on
FMR1 by $\mathrm{c}$ and $\mathrm{f}$ primers and detection of CGG repeat size by capillary electrophoresis were performed using a PCR based approach, which utilizes a chimeric primer, as previously described. ${ }^{27-29}$

\section{Southern blot confirmation}

Upon identification of a premutation allele, a peripheral blood sample was collected to confirm molecular diagnosis, from the proband and consenting extended family members. Genomic DNA was isolated and CGG repeat size and methylation status were determined using Southern blot and PCR analysis as previously described. ${ }^{26}$

\section{AGG interruption assay}

The position and number of AGG interruptions were determined for each allele by capillary electrophoresis as recently described. ${ }^{23,24,27}$ Briefly, amplification of the CGG repeats occur by variable annealing of the chimeric primer, where five CGG repeats are consecutive in sequence and addition of nucleotides through the remaining CGG repeat element and towards the $\mathrm{f}$ primer. Fragments corresponding to each length between the first 
CGG repeat and that of the fifth to the last repeat are made. The lack of PCR fragments in five consecutive repeat sizes indicates the presence of AGG interruptions. Using this characteristic of the triple primer PCR, the total length of the CGG repeat and the position of AGG interruptions along the repeat tract can be determined for both alleles (Figure 1). We assayed three of the DNA samples with the complementary triple primer PCR that amplifies using the same technique but in the opposite direction, in order to visualize a CGG repeat stretch comigrating with the $\mathrm{c}$ and $\mathrm{f}$ amplicon of the normal allele and subsequently masked by the larger peak in the electropherogram (Figure 1b).

\section{Haplotype markers}

Three microsatellite markers and two single-nucleotide polymorphisms (SNPs) were analyzed for all samples collected. Two of the microsatellite markers have complex polymorphisms. DXS548 is located approximately $157 \mathrm{~kb}$ upstream of the CGG repeat element, it contains a CA dinucleotide repeat and an indel. The analysis of this marker included two PCRs, the reverse primer (5'-AGAGCTTCACTATGCAATGGAATC) remained consistent and the forward primers annealed either upstream (DXS548-short) (5' [6-FAM]-GAATA GTCTCTGGGGTGGATCTC) or downstream (DXS548-long) (5' [6-FAM]GTACATTAGAGTCACCTGTGGTGC) of the indel. FRAXAC1 (5'-GATCTAA TCAACATCTATAGACTTTATT and 5' GATGAGAGTCACTTGAAGCTGG) is a GT dinucleotide repeat that is approximately $7 \mathrm{~kb}$ upstream of the CGG repeat element. FRAXAC2 (5'-GACTGCTCCGGAAGTTGAATCCTC A and 5'-CT AGGTGACAGAGTGAGATCCTGTC) is $11 \mathrm{~kb}$ downstream of the CGG repeat element, and includes a GT dinucleotide repeat followed by a TA dinucleotide repeat and a stretch of thymines with variable length. PCR of $100 \mathrm{ng}$ of genomic DNA was performed using Amplitaq Gold polymerase with Buffer II, $2.5 \mathrm{~mm} \mathrm{MgCl}, 200 \mu \mathrm{m}$ of each dNTP and $0.2 \mu \mathrm{m}$ of each primer. Thermal cycling conditions were $95^{\circ} \mathrm{C}$ for $7.5 \mathrm{~min}, 10$ cycles of $98^{\circ} \mathrm{C}$ for $30 \mathrm{~s}, 60^{\circ} \mathrm{C}$ for $1.5 \mathrm{~min}, 72^{\circ} \mathrm{C}$ for $1.5 \mathrm{~min} ; 22$ cycles of $98^{\circ} \mathrm{C}$ for $30 \mathrm{~s}, 55^{\circ} \mathrm{C}$ for $1.5 \mathrm{~min}, 72^{\circ} \mathrm{C}$ for $1.5 \mathrm{~min}$; and a final extension for $10 \mathrm{~min}$ at $72^{\circ} \mathrm{C}$. Additionally, two SNPs downstream of the CGG repeat element (rs4949 and rs25714) were analyzed using Taqman SNP genotyping following the manufacturer protocol.

\section{Reverse transcription-PCR}

Blood was collected in Tempus vacutainers from 16 of the participants and total RNA was extracted. Reverse transcription of mRNA was performed and FMR1 mRNA expression levels were measured by quantitative reverse transcription-PCR. Total RNA isolation and quantification of the FMR1 transcript levels were as previously described. ${ }^{12}$

\section{Clinical evaluation}

Individuals with a premutation size allele were clinically evaluated at the MIND Institute. Family medical history was collected along with clinical exams. Young children were administered developmental assessments, including the Vineland II Adaptive Behavior Composite and The Mullen Early Learning Composite. Older participants were administered tests to determine their cognitive abilities, motor skills, and symptoms related to fragile $\mathrm{X}$-associated disorders. Individual II-5 was also assessed using brain magnetic resonance imaging.

\section{RESULTS}

The male newborn (proband) was identified as a premutation carrier with 68 CGG repeats in FMR1 through newborn screening. A blood sample was collected from the proband during the first follow up visit and family members who consented to participate in the study. PCR and Southern blot analyses were performed to determine CGG allele size and AGG interruptions for 23 individuals ( 15 females and 8 males), including the proband. Additionally, genotyping information for five polymorphic sites flanking the CGG repeat element was obtained for the 23 individuals.

Ten females were identified as premutation carriers by molecular testing, and an additional female was determined to be an obligate premutation carrier (IV-11) based on her father being a premutation carrier (III-12). Four males were identified as premutation carriers by molecular testing and an additional male was determined to likely be a premutation carrier based on the presence of a premutation allele and on the haplotype results in the three daughters of the individual (I-1). Thus, a total of 16 premutation carriers were identified in this family (14 by DNA testing and 2 inferred).

\section{Transmission of the premutation alleles}

Seven transmission events where the premutation allele was inherited were maternal and four were paternal. Four additional paternal transmissions of the premutation allele likely occurred, three from the male in the first generation to all three daughters (I-1 to II-1, II-2 and II-5) and one occurred from III-12 to IV-11 (the latter was not tested, but IV-11 is an obligated carrier). Instability between individual I-1 and at least one daughter during paternal transmission of the premutation allele occurred, resulting in two different premutation allele sizes in the second generation. An increase of one CGG repeat occurred during transmission of the allele from II-2 to III-8. Transmission of the allele from II-5 to III-12 and III-15 were stable but increased by 2 CGG repeats to III-16. In the third generation, the alleles are present as 63 and 65 CGG repeats. Transmissions from the third to the fourth generation include two stable transmissions, two transmissions that increased the allele size by 1 CGG repeat, and one transmission that increased the allele size by 2 CGG repeats. In the fourth generation allele sizes ranged from 63 to 67 CGG repeats. Transmissions from the fourth to the fifth generation both included instabilities, an increase of 1 and 2 CGG repeats. The alleles observed in the fifth generation were 67 and 68 CGG repeats. Thus, the instability of the premutation allele through the five generations was a total of 6 CGG repeats.

\section{AGG interruption patterns}

Two AGG interruptions were present in all 14 premutation alleles. The position of the AGG interruptions was the same for all 14 premutation alleles, specifically in positions 10 and 20 (9-A-9-A-n). In addition, distribution patterns were variable in the alleles with a normal size range. A total of 11 different AGG interruption patterns were present in the normal alleles, with 10-A-9-A-9 observed at the highest frequency ( 9 out of 24 normal alleles). The majority of normal alleles had the first AGG interruption in position 10 or 11, but were also observed in positions 14,15 and 21 . Sixteen different AGG interruption patterns and 14 different CGG repeat lengths were observed between both the 24 normal and 14 premutation alleles (Table 1)

\section{Haplotypes}

The DXS548 marker had 3 observed alleles for the CA repeat, 20 chromosomes had 20 CA repeats, 2 chromosomes had 21 repeats, and 16 chromosomes had 25 repeats. The DXS548 marker also contained an indel downstream of the CA repeat; only chromosomes with 25 CA repeats had an insertion. Three alleles were observed for the FRAXAC1 marker, 18, 19 and 21. Three alleles were also observed for the FRAXAC2 marker, 151, 152 and 153. Both the G and A alleles were observed for SNP rs4949, but only the C allele was observed for the remaining SNP (rs25714). The haplotype for the premutation allele and the normal allele with an AGG pattern 9-A-9-A-13 were the same $(25+-21-C G G$ element-153-G-C). A summary of the haplotypes determined in the 23 subjects tested is shown in Table 1. 


\section{FMR1 expression}

FMR1 mRNA was measured in peripheral blood of 16 participants, 10 carriers for the premutation allele, and of these 9 were females. The FMR1 expression levels of the premutation carriers were between 1.06 and 1.99 (s.e. between 0.01 and 0.23). Six individuals had the normal range alleles (four females and two males) and their FMR1 expression levels varied between 1.06 and 1.42 (s.e. between 0.06 and 0.14 ). ${ }^{12}$ FMR1 mRNA levels were measured in the peripheral blood of three females who reported symptoms that are common in premutation carriers and all of them, II-2, IV-18 and IV-19, were found to be higher than what was observed in individuals who were not premutation carriers $(1.58$, s.e. $=0.02,1.99$, s.e. $=0.23$ and 1.81 , s.e. $=0.08$, respectively)

\section{Clinical evaluations}

The newborn and the family members, all Caucasian, carrying a premutation allele were evaluated clinically. Many premutation carrier individuals did not have symptoms characteristic of either FXTAS or

Table 1 AGG interruption patterns and haplotypes observed in family

\begin{tabular}{lccl}
\hline AGG pattern & CGG total length & Number of alleles & Haplotype \\
\hline $10-A-9$ & 20 & 2 & $21-19-C G G-152-A-C$ \\
$13-A-9$ & 23 & 2 & $20-19-C G G-151-G-C$ \\
$14-A-9$ & 24 & 1 & $20-19-C G G-151-G-C$ \\
$20-A-9$ & 30 & 1 & $20-19-C G G-152-A-C$ \\
$10-A-20$ & 31 & 1 & $20-19-C G G-152-A-C$ \\
$9-A-9-A-9$ & 29 & 2 & $20-19-C G G-151-G-C$ \\
$10-A-9-A-9$ & 30 & 9 & $20-19-C G G-152-A-C$ \\
$10-A-9-A-10$ & 31 & 3 & $20-19-C G G-151-A-C$ \\
$9-A-11-A-10$ & 32 & 1 & $20-18-C G G-153-U-U$ \\
$9-A-9-A-13$ & 33 & 2 & $25+-21-C G G-153-G-C$ \\
$9-A-9-A-42$ & 62 & 2 & $25+-21-C G G-153-G-C$ \\
$9-A-9-A-43$ & 63 & 5 & $25+-21-C G G-153-G-C$ \\
$9-A-9-A-44$ & 64 & 2 & $25+-21-C G G-153-G-C$ \\
$9-A-9-A-45$ & 65 & 2 & $25+-21-C G G-153-G-C$ \\
$9-A-9-A-47$ & 67 & 2 & $25+-21-C G G-153-G-C$ \\
$9-A-9-A-48$ & 68 & 1 & $25+-21-C G G-153-G-C$ \\
\hline
\end{tabular}

Abbreviaton: U: allele undetermined.
FXPOI, including tremor, gait ataxia, ${ }^{7}$ immuno-mediated disorders, ${ }^{30}$ hypertensions $^{31}$ and sleep apnea. ${ }^{32}$ The mother (IV-19) of the newborn reported having ptosis in the left eye and a brain aneurism at around 6 months gestation with the proband. Individual II-5, a premutation carrier female with 63 CGG repeats, was diagnosed with Alzheimer's disease before participating in this study. She presented with significant white matter disease in the frontal and parietal regions of her brain along with the insula bilaterally, decreased cognitive abilities starting at 81 years of age and movement problems that included difficulty walking in tandem with someone, unintelligible handwriting and a subtle tremor with positioning. Her symptoms were consistent with probable FXTAS. The two other female premutation carriers in generation II, II-1 and II-2 were also clinically evaluated. Individual II-1, 92 years old, had no symptoms common with FXTAS or FXPOI. Individual II-2, 84 years old, had a medical history that included cardiac problems (congestive heart failure, coronary artery bypass surgery and arrhythmias), resulting in the use of a pacemaker (common in aging carriers), as well as diabetes type II and bladder incontinence, also common in carriers. Two female premutation carriers in generation IV had panic attacks (IV-11) or sleep disorders (IV-18), symptoms common in the general population, but also frequently observed in premutation carriers. The proband identified by newborn screening did not show signs of clinical involvement pertaining to the premutation allele. At 6 months of age the proband scored 119 on the Vineland II Adaptive Behavior Composite and 105 on the Early Learning CompositeMullen test. More details involving the clinical symptoms of this family have been described. ${ }^{33}$

\section{DISCUSSION}

Peripheral whole blood was collected from 23 individuals belonging to the extended family of a newborn carrying a premutation allele identified through a newborn screening for fragile $\mathrm{X}$ study. The lineage for the premutation sized allele was traced to the newborn's mother's paternal grandmother's father and the size of the allele was followed through four generations as shown by pedigree chart (Figure 2) by DNA testing.

Small instabilities in premutation allele length were observed with all instabilities represented by an increase in size of one or two CGG

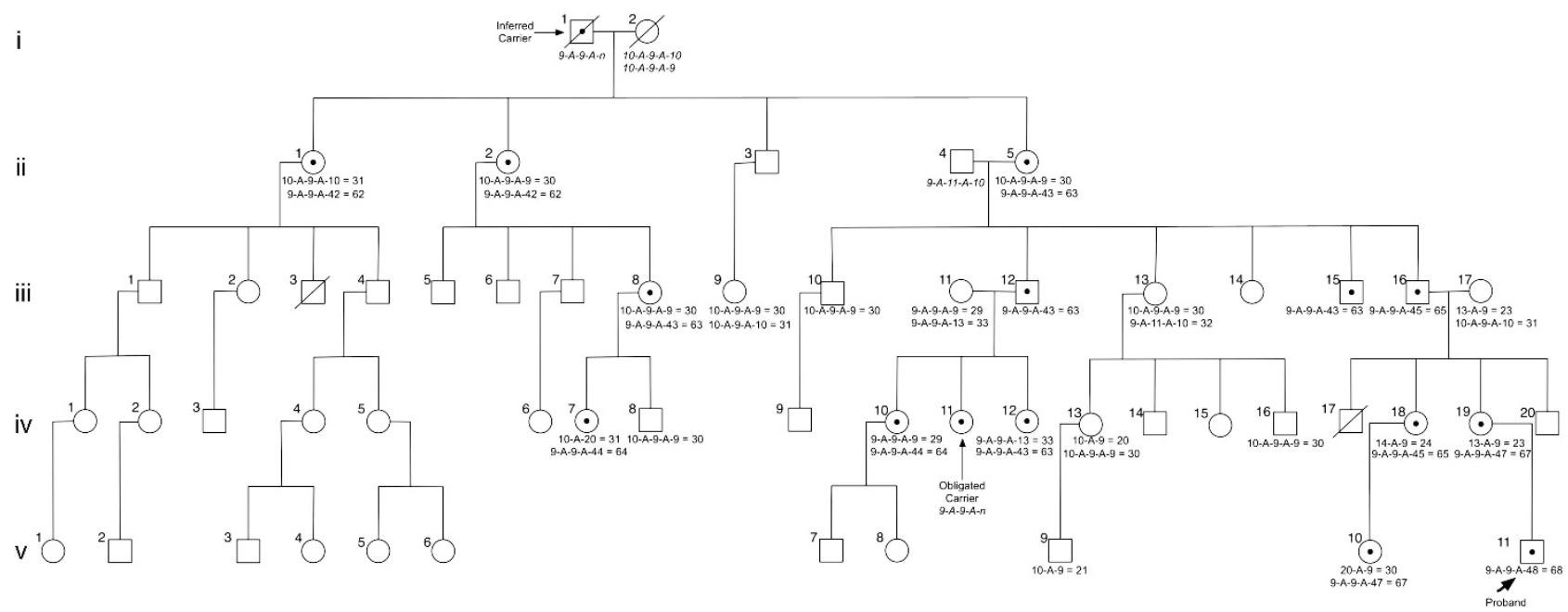

Figure 2 Family pedigree. The premutation allele is traced through five generations of the pedigree along with the haplotypes of markers adjacent to the CGG repeat element. The premutation allele has undergone instability through generations (range 0-6 CGG repeats). Several individuals were not available for DNA testing. Inferred AGG interruption patterns are italicized. 

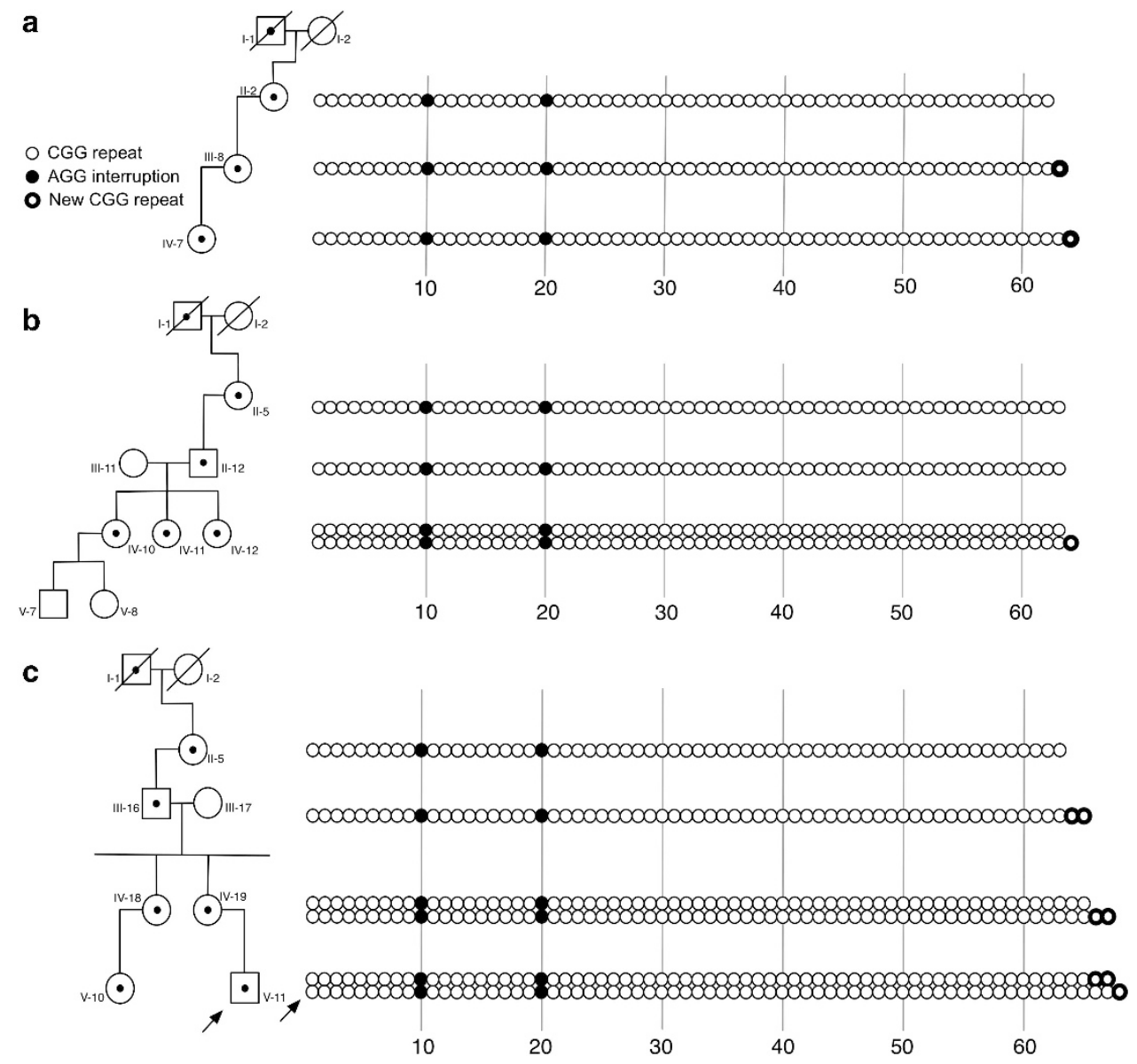

Figure 3 Difference in the CGG repeat is observed along multiple branches of the family pedigree. CGG repeat length is observed to increase down multiple branches of the pedigree, (a) from I-1 to IV-7 an increase of 2 CGG repeats, (b) from I-1 to IV-10, IV-11 and IV-12 an increase of 1 CGG repeat and (c) from $\mathrm{I}-1$ to $\mathrm{V}-10$ and $\mathrm{V}-11$ an increase of five CGG repeats.

repeats during transmission, for a maximum of six CGG repeats, and were observed in eight transmissions. Instabilities were present for both the maternal and paternal transmissions of the premutation allele. Additionally, the three siblings in generation II had two different premutation sizes, 62 and 63 CGG repeats, suggesting instability for at least one transmission between generations I and II. In generation II, premutation alleles were present in all three females. Additionally, two AGG patterns were observed for the normal alleles inherited by these three females, 10-A-9-A-9 and 10-A-9-A-10, each pattern also differed for the DXS548 microsatellite markers. Taken together, the inheritance of the three different haplotype blocks supports the male in generation I as being a carrier of the premutation allele. DNA was not analyzed for the male (II-3) in generation II, but would only have inherited a normal allele from his mother (I-2).

There were two AGG interruptions in every premutation allele with the pattern (CGG) ${ }_{9} \mathrm{AGG}(\mathrm{CGG})_{9} \mathrm{AGG}(\mathrm{CGG})_{42+}$ (Figure 3). The instability consistently occurred at the $3^{\prime}$ end of the repeat locus, in agreement with previous findings of CGG repeat expansion, which show the longer pure stretch of CGG repeats to be on the $3^{\prime}$ end in premutation alleles and expansion to occur at these longer stretches. ${ }^{23,34,35}$ No expansion to a full mutation was observed in any of the transmissions. Given the total CGG length and the number of AGG interruptions present in the FMR1 premutation allele in this family, this observation is consistent with the calculated risk of transmission to a full mutation, as predicted by Yrigollen et al., ${ }^{23}$ ranging from 0.6 to $2.0 \%$. Alleles in the range of $60-64$ CGG repeats with two AGG interruptions have been reported to be unstable during approximately $55.6 \%$ of transmissions and change in length by 1-24 CGG repeats; alleles within 65-69 CGG repeats with two AGG interruptions were observed to be unstable in $60 \%$ of the transmissions and increased or decreased by 1-4 CGG repeats during the transmission. ${ }^{36}$ In none of the transmissions was there any change in position of AGG interruptions.

Instability during transmission in three females through three generations showed two transmission events that each resulted in an additional CGG repeat, the total length of 62 CGG repeats in II-2 expanded to 63 CGG repeats in III-8 and to 64 CGG repeats in IV-7 (Figure 3a). We also observed a male with 63 CGG repeats (III-12) who transmitted the premutation allele to three daughters, one allele remained 63 CGG repeats (IV-12), one expanded by one repeat to 64 CGG repeats (IV-10) and for one daughter did not have DNA collected and was not analyzed (IV-11) (Figure 3b). Additionally, individual II-5 (63 CGG repeats) transmitted the premutation allele to three sons and the normal allele to two daughters and a son. Two of the sons who inherited the premutation allele showed no instability during transmission and one showed the allele increased by 2 CGG repeats to 65 CGG (III-16). Individual III-16 subsequently transmitted the premutation allele to 2 daughters, IV-18 (65 CGG repeats) and IV-19 (67 CGG repeats). Each of these daughters transmitted the 
premutation allele to 1 child resulting in expansion of 1 CGG repeat (IV-19 to V-11) and 2 repeats (IV-18 to V-10) (Figure 3c).

The results of the transmission events that have occurred within five generations in the identified family are consistent with the slow mutation pathway proposed by Eichler et al. ${ }^{37,38}$ The slow progression from a normal allele to a full-mutation allele is thought to occur when AGG interruptions remain intact in the CGG repeat locus, and expansions are polar (occur at the $3^{\prime}$ end of the locus) in small incremental steps. The stability of the locus is greater than, if one or both interruptions were absent resulting in a larger pure CGG repeat stretch. The DXS548, FRAXAC1 and FRAXAC2 haplotype observed in the premutation alleles are consistent with the 2-1-3 haplotype that was previously reported (described as $25-21-153$ in this study). ${ }^{37,38}$ This haplotype was associated with premutation alleles that maintain the two AGG interruption pattern and gradually increase in CGG repeat length.

Upon clinical evaluation, some members of the family were shown to have symptoms common in individuals with a premutation allele. Most notably, II-5 had the symptoms associated with FXTAS and had previously been diagnosed with Alzheimer's disease. It is likely that both conditions are occurring, which have been shown to be the case in neuropathological studies of eight females with the premutation who presented with cognitive decline in aging. ${ }^{39}$ Although observed in the general population, the additional symptoms of panic disorder, sleep disturbance, incontinence and heart problems are more common among premutation carriers during aging. 6,40,41

FMR1 expression level was not measured for II-5, however two of the three premutation carriers who reported health problems that may be associated with their carrier status had elevated FMR1 mRNA levels in the peripheral blood. The majority of participants with measured expression levels were females. FMR1 gene expression in females depends on the activation ratio, which defines the proportion of premutation allele on the active $\mathrm{X}$ chromosome, this confounds the FMR1 mRNA measurements more than when comparing mRNA levels in males. It is unclear whether the individuals with higher mRNA levels will be more likely to develop symptoms associated with premutation carrier status, although elevation of mRNA levels have been positively correlated with symptoms in premutation carriers. ${ }^{6,39,42}$

The majority of individuals identified as premutation carriers in this cascade testing study were younger than 60 years old. The age of onset for many of the late onset neurodegenerative disorders and symptoms associated with the premutation allele generally occur after the carrier is 60 years old. The premutation carriers identified are not certain to develop fragile $\mathrm{X}$-associated disorder symptoms; however, knowing their CGG size and number of the AGG interruptions within the FMR1 allele may improve how quickly they are diagnosed if symptoms begin and influence the treatment or decisions regarding reproductive choices.

Importantly, to our knowledge, this is the first transmission study of the FMR1 premutation allele through five generations in a family identified in the general population through a newborn screening pilot project. $^{29}$ Our previous transmission studies, showing the influence of the AGG interruption on allele stability, were carried out from a single generation in mother premutation carriers recruited through their child having a premutation or a full-mutation allele. ${ }^{23,36}$ In this study, we asked whether a premutation allele detected in the general population, rather than being recruited because of the presence of a subject with FXS in the family, would have behaved similarly and its stability being affected by the presence of AGG interruptions and CGG length. Our findings indicate that even in an unbiased sample from the general population, the risk of transmission to a full mutation follows the prediction reported by Yrigollen et al. ${ }^{23}$

Finally, detecting the presence of AGG interruptions within an FMR1 allele will provide an additional tool in genetic counseling for predicting the risk to expansion during transmission from a premutation to a full-mutation allele. ${ }^{23,36}$

\section{CONFLICT OF INTEREST}

FT is a nonpaid collaborator with Asuragen Inc. She has a patent for the detection of FMR1 allele size and category using the CGG linker PCR based approach. RH has received funding for treatment trials in fragile X syndrome or autism from Novartis, Roche, Seaside Therapeutics, Curemark, Forest Pharmaceuticals and the National Fragile X Foundation. CMY and GM-M have no conflicts of interest to disclose.

\section{ACKNOWLEDGEMENTS}

We thank the participating family for their support of this work. This work was supported by the National Institutes of Health (NIH) through individual research awards (HD02274, HD36071) and ARRA suppl. 3P30-HD00227442S2. The funders had no role in the study design, data collection and analysis, decision to publish or preparation of the manuscript. This work is dedicated to the memory of Matteo.

1 Lacoux, C., Di Marino, D., Boyl, P. P., Zalfa, F., Yan, B., Ciotti, M. T. et al. BC1-FMRP interaction is modulated by $2^{\prime}$-O-methylation: RNA-binding activity of the tudor domain and translational regulation at synapses. Nucleic. Acids Res. 40, 4086-4096 (2012).

2 Oostra, B. A. \& Willemsen, R. FMR1: a gene with three faces. Biochim. Biophys. Acta. 1790, 467-477 (2009).

3 Rooms, L. \& Kooy, R. F. Advances in understanding fragile $X$ syndrome and related disorders. Curr. Opin. Pediatr. 23, 601-606 (2011).

4 Chonchaiya, W., Schneider, A. \& Hagerman, R. J. Fragile X: a family of disorders. Adv. Pediatr. 56, 165-186 (2009).

5 Chonchaiya, W., Au, J., Schneider, A., Hessl, D., Harris, S. W., Laird, M. et al. Increased prevalence of seizures in boys who were probands with the FMR1 premutation and co-morbid autism spectrum disorder. Hum. Genet. 131, 581-589 (2012).

6 Bourgeois, J. A., Seritan, A. L., Casillas, E. M., Hessl, D., Schneider, A., Yang, Y. et al. Lifetime prevalence of mood and anxiety disorders in fragile $\mathrm{X}$ premutation carriers. J. Clin Psychiatry 72, 175-182 (2011).

7 Tassone, F. \& Berry-Kravis, E. M. Fragile X-associated tremor ataxia syndrome (FXTAS) (Springer, New York, NY, USA, 2010).

8 Pieretti, M., Zhang, F. P., Fu, Y. H., Warren, S. T., Oostra, B. A., Caskey, C. T. et al. Absence of expression of the FMR-1 gene in fragile X syndrome. Cell 66, 817-822 (1991)

9 Fu, Y. H., Kuhl, D. P. A., Pizzuti, A., Pieretti, M., Sutcliffe, J. S., Richards, S. et al. Variation of the Cgg repeat at the fragile-X site results in genetic instability-resolution of the sherman paradox. Cell 67, 1047-1058 (1991).

10 Garcia-Arocena, D. \& Hagerman, P. J. Advances in understanding the molecular basis of FXTAS. Hum. Mol. Genet. 19, R83-R89 (2010).

11 Hagerman, R. J., Leehey, M., Heinrichs, W., Tassone, F., Wilson, R., Hills, J. et al. Intention tremor, parkinsonism, and generalized brain atrophy in male carriers of fragile X. Neurology 57, 127-130 (2001).

12 Tassone, F., Hagerman, R. J., Taylor, A. K., Gane, L. W., Godfrey, T. E. \& Hagerman, P. J. Elevated levels of FMR 1 mRNA in carrier males: a new mechanism of involvement in the fragile-X syndrome. Am. J. Hum. Genet. 66, 6-15 (2000).

13 Kenneson, A., Zhang, F., Hagedorn, C. H. \& Warren, S. T. Reduced FMRP and increased FMR1 transcription is proportionally associated with CGG repeat number in intermediate-length and premutation carriers. Hum. Mol. Genet. 10, 1449-1454 (2001).

14 Allen, E. G., Sherman, S., Abramowitz, A., Leslie, M., Novak, G., Rusin, M. et al. Examination of the effect of the polymorphic CGG repeat in the FMR1 gene on cognitive performance. Behav. Genet. 35, 435-445 (2005).

15 Sellier, C., Rau, F., Liu, Y., Tassone, F., Hukema, R. K., Gattoni, R. et al. Sam68 sequestration and partial loss of function are associated with splicing alterations in FXTAS patients. EMBO J. 29, 1248-1261 (2010).

16 Jin, P., Duan, R., Qurashi, A., Qin, Y., Tian, D., Rosser, T. C. et al. Pur alpha binds to rCGG repeats and modulates repeat-mediated neurodegeneration in a Drosophila model of fragile $X$ tremor/ataxia syndrome. Neuron 55, 556-564 (2007).

17 Sofola, O. A., Jin, P., Qin, Y., Duan, R., Liu, H., de Haro, M. et al. RNA-binding proteins hnRNP $A 2 / B 1$ and CUGBP1 suppress fragile $X$ CGG premutation repeat-induced neurodegeneration in a Drosophila model of FXTAS. Neuron 55, 565-571 (2007). 
18 Hagerman, P. J. \& Hagerman, R. J. The fragile-X premutation: a maturing perspective. Am. J. Hum. Genet. 74, 805-816 (2004).

19 Verkerk, A. J., Pieretti, M., Sutcliffe, J. S., Fu, Y. H., Kuhl, D. P., Pizzuti, A et al. Identification of a gene (FMR-1) containing a CGG repeat coincident with a breakpoint cluster region exhibiting length variation in fragile $\mathrm{X}$ syndrome. Cell 65 , 905-914 (1991).

20 Eichler, E. E. \& Nelson, D. L. Genetic variation and evolutionary stability of the FMR1 CGG repeat in six closed human populations. Am. J. Med. Genet. 64, 220-225 (1996).

21 Arrieta, I., Telez, M., Huerta, I., Flores, P., Criado, B., Ramirez, J. M. et al. Fragile X gene stability in Basque Valleys: prevalence of premutation and intermediate alleles. Hum. Biol. 80, 593-600 (2008).

22 Eichler, E. E., Holden, J. J., Popovich, B. W., Reiss, A. L., Snow, K., Thibodeau, S. N. et al. Length of uninterrupted CGG repeats determines instability in the FMR1 gene. Nat. Genet. 8, 88-94 (1994).

23 Yrigollen, C. M., Durbin-Johnson, B., Gane, L., Nelson, D. L., Hagerman, R., Hagerman, P. J. et al. AGG interruptions within the maternal FMR1 gene reduce the risk of offspring with fragile X syndrome. Genet. Med. 14, 729-736 (2012).

24 Yrigollen, C. M., Tassone, F., Durbin-Johnson, B. \& Tassone, F. The role of AGG interruptions in the transcription of FMR1 premutation alleles. PLOS One 6, e21728 (2011).

25 Ludwig, A. L., Raske, C., Tassone, F., Garcia-Arocena, D., Hershey, J. W. \& Hagerman, P. J. Translation of the FMR1 mRNA is not influenced by AGG interruptions. Nucleic. Acids. Res. 37, 6896-6904 (2009).

26 Tassone, F., Pan, R., Amiri, K., Taylor, A. K. \& Hagerman, P. J. A rapid polymerase chain reaction-based screening method for identification of all expanded alleles of the fragile X (FMR1) gene in newborn and high-risk populations. J. Mol. Diagn. 10, 43-49 (2008).

27 Chen, L., Hadd, A., Sah, S., Filipovic-Sadic, S., Krosting, J., Sekinger, E. et al. An information-rich CGG repeat primed PCR that detects the full range of fragile $X$ expanded alleles and minimizes the need for southern blot analysis. J. Mol. Diagn. 12, 589-600 (2010).

28 Filipovic-Sadic, S., Sah, S., Chen, L., Krosting, J., Sekinger, E., Zhang, W. et al. A novel FMR1 PCR method for the routine detection of low abundance expanded alleles and full mutations in fragile X syndrome. Clin. Chem. 56, 399-408 (2010).

29 Tassone, F., Long, K. P., Tong, T. H., Lo, J., Gane, L. W., Berry-Kravis, E. et al. FMR1 CGG allele size and prevalence ascertained through newborn screening in the United States. Genome. Med. 4, 100 (2012).

30 Winarni, T. I., Chonchaiya, W., Sumekar, T. A., Ashwood, P., Morales, G. M., Tassone, F. et al. Immune-mediated disorders among women carriers of fragile $\mathrm{X}$ premutation alleles. Am. J. Med. Genet. A. 158A, 2473-2481 (2012).
31 Hamlin, A. A., Sukharev, D., Campos, L., Mu, Y., Tassone, F., Hessl, D. et al. Hypertension in FMR1 premutation males with and without fragile X-associated tremor/ ataxia syndrome (FXTAS). Am. J. Med. Genet. A. 158A, 1304-1309 (2012).

32 Hamlin, A., Liu, Y., Nguyen, D. V., Tassone, F., Zhang, L. \& Hagerman, R. J. Sleep apnea in fragile X premutation carriers with and without FXTAS. Am. J. Med. Genet. B. Neuropsychiatr. Genet. 156B, 923-928 (2011).

33 Sorensen, P. L., Gane, L. W., Yarborough, M., Hagerman, R. J. \& Tassone, F. Newborn screening and cascade testing for FMR1 mutations. Am. J. Med. Genet. A. 161, 59-69 (2013).

34 Eichler, E. E., Kunst, C. B., Lugenbeel, K. A., Ryder, O. A., Davison, D., Warren, S. T. et al. Evolution of the cryptic FMR1 CGG repeat. Nat. Genet. 11, 301-308 (1995).

35 Eichler, E. E., Hammond, H. A., Macpherson, J. N., Ward, P. A. \& Nelson, D. L. Population survey of the human FMR1 CGG repeat substructure suggests biased polarity for the loss of AGG interruptions. Hum. Mol. Genet. 4, 2199-2208 (1995).

36 Nolin, S. L., Sah, S., Glicksman, A., Sherman, S., Allen, E., Berry-Kravis, E. et al. Fragile X AGG analysis provides new risk predictions for 45-69 repeat alleles. Am. J. Med. Genet. A. 161, 771-778 (2013).

37 Eichler, E. E., Macpherson, J. N., Murray, A., Jacobs, P. A., Chakravarti, A. \& Nelson, D. L. Haplotype and interspersion analysis of the FMR1 CGG repeat identifies two different mutational pathways for the origin of the fragile X syndrome. Hum. Mol. Genet. 5, 319-330 (1996).

38 Larsen, L. A., Armstrong, J. S., Gronskov, K., Hjalgrim, H., Macpherson, J. N., Brondum-Nielsen, $\mathrm{K}$. et al. Haplotype and AGG-interspersion analysis of FMR1 $(\mathrm{CGG})(\mathrm{n})$ alleles in the Danish population: implications for multiple mutational pathways towards fragile X alleles. Am. J. Med. Genet. 93, 99-106 (2000).

39 Tassone, F., Greco, C. M., Hunsaker, M. R., Seritan, A. L., Berman, R. F. Gane, L. W. et al. Neuropathological, clinical and molecular pathology in female fragile $X$ premutation carriers with and without FXTAS. Genes. Brain. Behav. 11, 577-585 (2012).

40 Chonchaiya, W., Nguyen, D. V., Au, J., Campos, L., Berry-Kravis, E. M., Lohse, K. et al. Clinical involvement in daughters of men with fragile $\mathrm{X}$-associated tremor ataxia syndrome. Clin. Genet. 78, 38-46 (2010).

41 Hunsaker, M. R., Greco, C. M., Tassone, F., Berman, R. F., Willemsen, R., Hagerman, R. J. et al. Rare intranuclear inclusions in the brains of 3 older adult males with fragile $x$ syndrome: implications for the spectrum of fragile $x$-associated disorders. $J$. Neuropathol. Exp. Neurol. 70, 462-469 (2011).

42 Tassone, F., Hagerman, R. J., Taylor, A. K., Mills, J. B., Harris, S. W., Gane, L. W. et al. Clinical involvement and protein expression in individuals with the FMR1 premutation. Am. J. Med. Genet. 91, 144-152 (2000). 